\title{
Comparison of the New Estimators: The Semi-Parametric Likelihood Estimator, SPW, and the Conditional Weighted Pseudo Likelihood Estimator, WPCE
}

\author{
Samuel Joel Kamun ${ }^{1}$, Richard Simwa ${ }^{2}$, Stanley Sewe ${ }^{1}$ \\ ${ }^{1}$ Department of Mathematics and Computing Studies, Faculty of Science, Catholic University of Eastern Africa, Nairobi, Kenya \\ ${ }^{2}$ Department of Mathematics, Faculty of Science, University of Nairobi, Nairobi, Kenya \\ Email address: \\ samuelkamun@gmail.com(S. J. Kamun), richardsimwa@uonbi.ac.ke(R. Simwa), ssewew@cuea.edu (S. Sewe) \\ ${ }^{*}$ Corresponding author
}

To cite this article:

Samuel Joel Kamun, Richard Simwa, Stanley Sewe. Comparison of the New Estimators: The Semi-Parametric Likelihood Estimator, SPW, and the Conditional Weighted Pseudo Likelihood Estimator, WPCE. American Journal of Theoretical and Applied Statistics.

Vol. 10, No. 4, 2021, pp. 202-207. doi: 10.11648/j.ajtas.20211004.14

Received: August 6, 2021; Accepted: August 21, 2021; Published: August 31, 2021

\begin{abstract}
The analysis of sample-based studies involving sampling designs for small sample size, is challenging because the sample selection probabilities (as well as the sample weights) is dependent on the response variable and covariates. The study has focused on using systems of weighted regression estimating equations, using different modified weights, to estimate the coefficients of Weighted Likelihood Estimators. Usually, the design-consistent (Weighted) estimators are obtained by solving (sample) weighted estimating equations. They are then used to construct estimates which have better relative efficiencies and smaller finite small sample bias than the estimates from the Horvitz-Thompson Weighted Estimator with unmodified weight, option A. The purpose of our study is to compare derived Estimators of the weighted regression estimating equations for estimating the coefficients of Weighted Likelihood Estimators, the Semi-Parametric Weighted Likelihood Estimator, SPW and the Weighted Conditional Pseudo Likelihood Estimator, WCPE with the conventional Horvitz-Thompson Weighted Likelihood Estimator, using relative efficiency, sample bias and Standard Error for small sample size. The constructed estimates from the system of weighted regression estimating equations, using different modified weights, are actually the Weighted Likelihood Estimators. The study compared the two new estimators, the Semi-parametric weighted estimator, SPW and the Weighted Conditional Pseudo Likelihood estimator, WCPE, for both the unmodified and modified Weights, which were found to have better relative efficiency and smaller finite small sample bias than the estimates from conventional Horvitz-Thompson Weighted Estimator, for both generated and for real data. The outcome of the tests show strong similarity in performance to those obtained using the simulated data. Estimates were constructed which have better relative efficiencies and smaller finite small sample bias than the estimates from the Horvitz-Thompson Weighted Estimator with unmodified weight, option A.
\end{abstract}

Keywords: Semi Parametric, Imputation, Estimating Error, Small Samples, Estimators, Relative Efficiency, Sample Bias

\section{Introduction}

This study is a follow up on the main reference found in Kamun et al [22]. The study has focused on using systems of weighted regression estimating equations [14], using different modified weights, to estimate the coefficients of Weighted Likelihood Estimators [1, 3-5]. We use them to construct estimates which have better relative efficiencies and smaller finite small sample bias than the estimates from the Horvitz-Thompson Weighted Estimator with unmodified weight, option A. Further inference on Stratified samples can be found in [15].

\subsection{The Model}

Suppose data, Lawless et al. [18], is produced according to a function

$$
f(y \mid x ; \theta) g(x)
$$

where $\mathrm{y}$ is a response variable which is multivariate and $\mathrm{x}$ is 
a continuous or discrete vector of covariate variables and

$$
\mathrm{f}(\mathrm{y} \mid \mathrm{x} ; \theta)
$$

is the regression part of the function. The marginal distribution of $\mathrm{x}$ is denoted by $\mathrm{g}(\mathrm{x})$, which for this study we have used Gaussian density to represent, is as shown below.

$$
k(u)=\frac{1}{\sqrt{(2 \pi)}} e^{-\left(\frac{u^{2}}{2}\right)}
$$

where $u=\frac{x_{1}-\operatorname{mean}\left(x_{1}\right)}{s \tan \cdot \operatorname{dev}\left(x_{1}\right)}$

We describe the conditional distribution of $\mathrm{y}$ given $\mathrm{x}_{1}$ as $\theta$. The likelihood is given by

$$
\prod f(y \mid x ; \theta)
$$

\subsection{System of Estimating Equations}

Let the function below represents a regression equation

$$
y=f\left(y_{j} \mid x_{j} ; \theta\right) g\left(x_{j}\right)
$$

multiplying equation (5) by $\mathrm{x}_{\mathrm{j}}$

$$
x_{j} y=x_{j} f\left(y_{j} \mid x_{j} ; \theta\right) g\left(x_{j}\right)
$$

and summing from $\mathrm{j}=1$ to $\mathrm{N}$ equation (6)

$$
\sum_{j=1}^{N} x_{j} y=\sum_{j=1}^{N} x_{j} f\left(y_{j} \mid x_{j} ; \theta\right) g\left(x_{j}\right)
$$

We can rewrite the equation as

$$
\sum_{j=1}^{N} x_{j} y-\sum_{j=1}^{N} x_{j} f\left(y_{j} \mid x_{j} ; \theta\right) g\left(x_{j}\right)=0
$$

or as

$$
\sum_{j=1}^{N} x_{j}\left(y-f\left(y \mid x_{j}, \theta\right) g\left(x_{j}\right)\right)=0
$$

and when we multiply the equation by the weight $\mathrm{w}_{\mathrm{j}}$ it gives us the sample weighted estimating equations

$$
\sum_{j=1}^{N} w_{j} x_{j}\left(y-f\left(y \mid x_{j}, \theta\right) g\left(x_{j}\right)\right)=0
$$

the sample estimating equations [14] and sample weighted estimating equations have been used as equations (9) and (10) respectively and are used to find solutions to regression equations [13].

\section{Weighted Likelihood}

In our study, we propose two novel weighted system of regression estimating equation for estimating coefficients for small sample studies: the small sample pseudo likelihood weighted (SPW) model and the small sample semi parametric weighted conditional (WCPE) model [17]. By fitting a regression model to the establish sample weights against the sample variables and or response variable [7], which is the requirement for the two estimates to be obtained we end up estimating the conditional expectation of the weights.

We modified the original sample weights, for WCPE, where the estimated conditional expectation of the weights is used. For our study, both response variables and predictor variables were used to find estimated weights. The SPW and WCPE models are an improvement of the design-based weighted estimates with improvement achieved by using suitable modification on the original sample weights before the estimation (e.g., [8]).

\subsection{Horvitz-Thompson Weighted Estimator}

Consider a finite population $\Omega$ of $\mathrm{N}$ individuals. Let $\mathrm{y}$ denote the response variables and $\mathrm{v}\left(\mathrm{x}^{\prime}, \mathrm{z}^{\prime}\right)$ ' denote the vector of all measured covariates, where $x$ are covariates associated with the outcome and $\mathrm{z}$ are the sample variables used in the process of sample selection. $\mathrm{x}$ and $\mathrm{z}$ may or may not have common variables.

Let $\mathrm{n}$ be the size of the observed sample $\mathrm{S}$. The probability that individual $\mathrm{i}, \mathrm{i}=1,2,3, \ldots, \mathrm{n}$, is included in the sample is denoted by $\pi_{\mathrm{i}}$. The base sample weight $\mathrm{w}_{\mathrm{i}}$ is defined as a reciprocal of the sample inclusion probability $\pi \mathrm{i}$, so $\pi \mathrm{i}=1 / \mathrm{w}_{\mathrm{i}}$. We refer to the final sample weight as the sample weight of individual $\mathrm{i}$ and denoted by $\mathrm{w}_{\mathrm{i}}$.

We have assumed in this study that the observed data are the data for the simulated units, for individuals $i \varepsilon S, i=1$, $2, \ldots, n$., as we observe $\left(\mathrm{y}_{\mathrm{i}}, \mathrm{v}_{\mathrm{i}}{ }^{\prime}, \mathrm{w}_{\mathrm{i}}{ }^{\prime}\right)^{\prime}$. We have assumed also the availability of sample proportions and means for the variables in $\mathrm{z}_{\mathrm{i}}$, which can be used to calibrate the sample weights.

In the population, the outcomes $\mathrm{Y}_{\mathrm{i}}$ conditional on covariates $\mathrm{X}_{\mathrm{i}}$ have distribution

$$
f\left(Y_{i} \mid X_{i}, \theta\right) g\left(X_{i}\right)
$$

For our study we use

$$
f\left(Y_{i} \mid X_{i}, \theta\right)=\beta_{0}+\beta_{i} X_{i}^{\prime}
$$

Where $\beta_{i}=\left(\beta_{0}, \beta_{1}, \beta_{2}, \beta_{3}, \beta_{4}\right)$.

By fitting model (1) to the entire finite population $\Omega$, we can estimate $\beta_{\mathrm{i}}$ consistently by solving a system of estimating equations with respect to $b$ :

$$
G(b)=\sum_{i=1}^{N} x_{i}\left(y_{i}-f\left(y_{i} \mid x_{i}, \theta\right) g\left(x_{i}\right)\right)=0
$$

where

$$
f\left(y_{i} \mid x_{i}, \theta\right) g\left(x_{i}\right)=\beta_{0}+\beta_{1} x_{1}+\beta_{2} x_{2}+\beta_{3} x_{3}+\beta_{4} x_{4}
$$


and $\mathrm{g}\left(\mathrm{x}_{\mathrm{i}}\right)$ is the marginal distribution of $\mathrm{x}_{\mathrm{i}}$ obtained non parametrically. In equation (5), $\mathrm{G}(\mathrm{b})$ above, are the maximum likelihood estimating equations based on all the values $\left(\mathrm{y}_{\mathrm{i}}, \mathrm{x}_{\mathrm{i}}\right)$ $\varepsilon \Omega$ if $\Omega$ is a stratified random sample from a super population generated under model (1)(cluster sampling could equally be used [2]). Denote the solution of (5) by B.

For any fixed value of $B, G(B)$ is a vector of finite sample totals, and hence, it can be estimated from the sample $\mathrm{S}$ by

$$
\hat{G}_{w}(b)=\sum_{i \varepsilon S} w_{i} x_{i}\left(y_{i}-f\left(y_{i} \mid x_{i}, \theta\right) g\left(x_{i}\right)\right)
$$

where $y_{i}$ is the observed response for the unit $i$. We denote the solution of the system of the equations $\hat{G}_{w}(b)=0$ by $\hat{\beta}_{w}$ which is referred to as the weighted estimator (the Horvitz-Thompson estimator).

\subsection{Weighted Conditional Likelihood: Pseudo Maximum Likelihood Estimator}

Let $f_{p}\left(w_{i} \mid y_{i}, x_{i}, \theta\right)$, which is the conditional pdf for the weight in the population [10], be the weight of the population distribution where the observed weight stems from the sample distribution $\mathrm{f}_{\mathrm{s}}$, obtained by Bayes rule as

$$
f_{p}\left(w_{i} \mid y_{i}, v_{i}, \theta\right)=f_{p}\left(w_{i} \mid y_{i}, z_{i}, i \varepsilon S, \theta\right)=\frac{\operatorname{Pr}\left(i \varepsilon S \mid y_{i}, v_{i}\right) f_{p}\left(w_{i} \mid y_{i}, x_{i}, \theta\right)}{\operatorname{Pr}(i \varepsilon S)}
$$

We assume from equation (13) and in this study that

$$
f_{p}\left(w_{i} \mid y_{i}, v_{i}, \theta\right)=f_{p}\left(w_{i} \mid y_{i}, x_{i}, \theta\right)
$$

for each value of i. This is an example of informative sampling that should be accounted for in the inferential process.

Equation (13) can be written equivalently by

$$
f_{s}\left(w_{i} \mid y_{i}, v_{i}, \theta, \gamma, \beta\right)=\frac{E_{s}\left(w_{i} \mid \theta, \gamma, \beta\right) f_{p}\left(w_{i} \mid y_{i}, x_{i}, \theta, \beta\right)}{E_{s}\left(w_{i} \mid y_{i}, v_{i}, \gamma\right)}
$$

Where $E_{s}\left(w_{i} \mid.\right)$ refers to the conditional expectation of the weights $\mathrm{w}_{\mathrm{i}}$ with respect to their sample distribution. Also $\gamma$ is an unknown vector of the unknown regression coefficients of the regression model on the design variables and the outcome for sample weights [11].

$$
G_{S}\left(w_{i}, \gamma, \beta\right)=\sum_{i \varepsilon S} \frac{\partial E_{s}\left(w_{i} \mid y_{i}, v_{i}, \theta, \gamma, \beta\right)}{\partial \beta}
$$

is the sample log-likelihood score equation in relation to $\beta$.

It follows then from equation (10), if it can be assumed that (1) is a population distribution of $y_{i}$ given $x_{i}$, that these equations can equivalently be written as

$$
G_{S}\left(w_{i}, \gamma, \beta\right)=\sum_{i \varepsilon S} x_{i}\left(y_{i}-f\left(y_{i} \mid x_{i}, \theta\right) g\left(x_{i}\right)\right) * \sum_{i \varepsilon S} \frac{\partial E_{s}\left(w_{i} \mid y_{i}, v_{i}, \theta, \gamma, \beta\right)}{\partial \beta}
$$

or

$$
G_{S}\left(w_{i}, \gamma, \beta\right)=\frac{\sum_{i \varepsilon S} x_{i}\left(y_{i}-f\left(y_{i} \mid x_{i}, \theta\right) g\left(x_{i}\right)\right)}{\sum_{i \varepsilon S} \frac{\partial E_{s}\left(w_{i} \mid y_{i}, v_{i}, \theta, \gamma, \beta\right)}{\partial \beta}}
$$

Where $E_{s}\left(w_{i} \mid v_{i}, \theta, \beta, \gamma\right)$, can be differentiated with respect to $\beta$ for any fixed $\gamma$.

We estimate $\gamma$ separately, using individual-level data $\mathrm{w}_{\mathrm{i}}, \mathrm{y}_{\mathrm{i}}$, $\mathrm{v}_{\mathrm{i}}$ observed for sample units, $i \in \mathrm{S}$. We then express the sample expectations $E_{S}\left(w_{i} \mid v_{i}\right)$ as functions of $\beta$ and the estimated value of $\gamma$. We then obtain the system of equations from (15) or (16) by substituting $\hat{\gamma}$ in place of $\gamma$, and solve it iteratively with respect to $\beta$,

$$
G_{S}(\hat{\gamma}, \beta)=0
$$

We denote this solution out of (9 or 10) by $\hat{\beta}_{W C P E}$ and refer to it as an WCPE estimator according to [12].

\subsection{Semi-parametric Weighted Estimator}

We obtain the modified sample weights using four steps as follows: i. We obtain sample weights from two samples, $\mathrm{n}$ and $\mathrm{g}$, where $\mathrm{w}_{\mathrm{g}}$ and $\mathrm{w}_{\mathrm{n}}$ are worked out as regression of the sample weights $\mathrm{w}_{\mathrm{i}}$ on $\mathrm{y}_{\mathrm{i}}, \mathrm{z}, \mathrm{x}_{1}, \mathrm{x}_{2}, \mathrm{x}_{3}$ and $\mathrm{x}_{4}$, for $\mathrm{w}_{\mathrm{g}}$ and $\mathrm{w}_{\mathrm{i}}$ on $\mathrm{x}_{1}, \mathrm{x}_{2}, \mathrm{x}_{3}$ and $\mathrm{x}_{4}$ for $\mathrm{w}_{\mathrm{n}}$.

ii. We then obtain sample weights from the two samples, $n$ and $\mathrm{g}$, where $\mathrm{w}_{\text {reg1 }}$ and $\mathrm{w}_{\text {reg2 }}$ are worked out as regression using least squares of sample weights $\mathrm{w}_{\mathrm{i}}$ on $\mathrm{y}_{\mathrm{i}}, \mathrm{z}, \mathrm{x}_{1}, \mathrm{x}_{2}, \mathrm{x}_{3}$ and $\mathrm{x}_{4}$ for $\mathrm{w}_{\mathrm{reg} 1}$ and $\mathrm{w}_{\mathrm{i}}$ on $\mathrm{x}_{1}, \mathrm{x}_{2}, \mathrm{x}_{3}$ and $\mathrm{x}_{4}$ for $\mathrm{w}_{\text {reg2 }}$.

iii. We define the weights that have been rescaled as $\tilde{w}_{i}$ by

$$
\tilde{w}_{i}=\left(\frac{w_{g}+w_{n}}{2}\right)
$$

iv. We then fit the regression model to the weight which have been rescaled, against the covariates $v_{i}$ and compute

$$
E_{S}\left(\tilde{w}_{i} \mid v_{i}\right)=E_{S}\left(\tilde{w}_{i} \mid z_{i}, \psi\right)
$$

Where $\psi$ refers to the estimated coefficients of the regression model which relates $\tilde{w}_{i}$ to the covariates $\mathrm{v}_{\mathrm{i}}$. We define the model with adjusted weights by 


$$
\tilde{w}_{i}^{m}=\frac{w_{\text {reg } 1}}{E\left(w_{\text {reg } 1} \mid x_{i}, y_{i}, z\right)} * \frac{E\left(w_{\text {reg } 2} \mid x_{i}\right)}{w_{\text {reg } 2}} * \frac{w_{i}}{E\left(w_{i} \mid x_{i}\right)}
$$

The system of weighted estimating equations

$$
\hat{G}_{S P W}=\sum_{i \varepsilon S} \tilde{w}_{i}^{m} x_{i}\left(y_{i}-f\left(y_{i} \mid x_{i}, \theta\right) g\left(x_{i}\right)\right)=0
$$

is solved with regards to $\hat{\beta}_{S P W}$, and its solution, is the semiparametric weighted estimator as proposed in [10].

\section{Results}

We repeated the simulations 10,000 times for sample plan A. We computed the simulated bias for the regression coefficients as the average of the difference of the estimated coefficient minus the true coefficient. We computed the simulated standard deviation for a regression coefficient by dividing the standard error by the number of simulations. We have used software packages designed for analysis of the weighted estimators using R [16].

\begin{tabular}{|c|c|c|c|c|c|c|c|c|}
\hline Estimators & Plan & $\begin{array}{l}\text { Coefficient of } \\
\text { Determination. } \mathbf{R}^{2}\end{array}$ & $\begin{array}{l}\text { Adjusted Coefficient } \\
\text { of Determination. } \mathbf{R}^{2}\end{array}$ & Bias & $\begin{array}{l}\text { Standard } \\
\text { Error }\end{array}$ & $\begin{array}{l}\text { Relative Efficiency } \\
\text { (Var1/Var2) }\end{array}$ & Mean & Stan.Dev \\
\hline \multirow[t]{3}{*}{ WCPE (MOM) } & $\mathrm{A}$ & 0.99999999997974 & 0.99999999997 & $2.5881519 \mathrm{e}-12$ & $1.2250786 \mathrm{e}-11$ & 0.982396 & 45.79445 & 0.51578 \\
\hline & $\mathrm{B}$ & 0.99999999997379 & 0.99999999996 & $4.5261572 \mathrm{e}-12$ & $1.4955370 \mathrm{e}-11$ & 1.045718 & 45.74961 & 0.49992 \\
\hline & $\mathrm{C}$ & 0.99999999998251 & 0.99999999998 & $2.8067548 \mathrm{e}-12$ & $9.623465 \mathrm{e}-12$ & 1.136823 & 45.70296 & 0.47947 \\
\hline \multirow[t]{3}{*}{ WCPE (MASS) } & A & 0.99999999998446 & 0.999999999978 & $4.2077453 e-12$ & $6.4815375 \mathrm{e}-12$ & 1.260005 & 45.76692 & 0.45543 \\
\hline & $\mathrm{B}$ & 0.99999999996874 & 0.999999999956 & $8.6316509 \mathrm{e}-12$ & $1.8434238 \mathrm{e}-11$ & 1.042005 & 45.74961 & 0.50081 \\
\hline & $\mathrm{C}$ & 0.99999999999274 & 0.99999999999 & $1.6465718 \mathrm{e}-12$ & $4.3478758 \mathrm{e}-12$ & 1.071496 & 45.77053 & 0.49387 \\
\hline \multirow[t]{3}{*}{ WCPE (STATS4) } & A & 0.99999999997703 & 0.999999999968 & $4.3728354 \mathrm{e}-12$ & $1.2832394 \mathrm{e}-11$ & 0.993460 & 45.76174 & 0.51290 \\
\hline & $\mathrm{B}$ & 0.99999999998055 & 0.999999999973 & $2.4388269 \mathrm{e}-12$ & $1.1426680 \mathrm{e}-11$ & 0.983769 & 45.76172 & 0.51542 \\
\hline & $\mathrm{C}$ & 0.99999999999922 & 0.999999999999 & $1.2534418 \mathrm{e}-13$ & $4.7164760 \mathrm{e}-13$ & 0.034135 & 45.62707 & 2.76699 \\
\hline \multirow[t]{3}{*}{ SPW } & A & 0.99999999994496 & 0.999999999923 & $8.9096508 \mathrm{e}-12$ & $4.4634743 \mathrm{e}-11$ & 2.695988 & 45.85448 & 0.31135 \\
\hline & $\mathrm{B}$ & 0.99999999996414 & 0.99999999995 & $8.5930152 \mathrm{e}-12$ & $1.5639812 \mathrm{e}-11$ & 0.993661 & 45.74964 & 0.51286 \\
\hline & $\mathrm{C}$ & 0.999999999985982 & 0.99999999998 & $3.2606140 \mathrm{e}-12$ & $7.6167599 \mathrm{e}-12$ & 1.152102 & 45.71676 & 0.47628 \\
\hline \multirow[t]{3}{*}{ HTWE } & A & 0.9999999999585073 & 0.999999999942 & $1.0464185 \mathrm{e}-11$ & $1.8758009 \mathrm{e}-11$ & 1.000000 & 45.77449 & 0.51122 \\
\hline & $\mathrm{B}$ & 0.999999999983550 & 0.999999999977 & $1.1735057 \mathrm{e}-12$ & $1.2060731 \mathrm{e}-11$ & 1.051093 & 45.74964 & 0.49864 \\
\hline & $\mathrm{C}$ & 0.999999999981308 & 0.999999999974 & $3.9779291 \mathrm{e}-12$ & $1.0356865 \mathrm{e}-11$ & 1.118182 & 45.72881 & 0.48345 \\
\hline HTWE & $\mathrm{w}=1$ & 0.9999999999844992 & 0.999999999978 & $3.1787906 \mathrm{e}-12$ & $9.2209949 \mathrm{e}-12$ & 1.046095 & 45.74960 & 0.49983 \\
\hline Monte Carlo & & 0.9999999999790133 & 0.999999999971 & $6.2848615 \mathrm{e}-12$ & $8.9509268 \mathrm{e}-12$ & 1.043087 & 45.74961 & 0.50055 \\
\hline
\end{tabular}

Table 1. Summary of Comparison and Analysis of Estimates for Generated Data.

HTWE $=$ Horvitz-Thompson Weighted Estimator (HTWE); SPW = Semi parametric Weighted Estimator,

$\mathrm{WCPE}=$ Weighted Conditional semi parametric Estimator., HTWE $(\mathrm{w}=1)=$ Un-weighted HTWE Estimator,

Var1 = Variance of HTWE Option A, Var2 = Variance of another Estimator.

The results in Table 1 show that all the Estimators for options A, B and C, except WCPE (STATS4) options A, B and C, with lower relative efficiency, have higher relative efficiencies and coefficients of determination than HTWE for option A including Monte Carlo Simulation, and hence are more efficient for Simulated Data for $\mathrm{n}=15$.

\begin{tabular}{|c|c|c|c|c|c|c|c|}
\hline Estimators & Plan & $\begin{array}{l}\text { Coefficient of } \\
\text { Determination. R2 }\end{array}$ & Bias & Standard Error & $\begin{array}{l}\text { Relative Efficiency } \\
\text { (Var1/Var2) }\end{array}$ & Mean & Stan.Dev \\
\hline \multirow[t]{3}{*}{ WCPE (MOM) } & $\mathrm{A}$ & 0.999999999910297 & $2.0048 \mathrm{e}-11$ & $4.786 \mathrm{e}-11$ & 0.8759 & 116.2194 & 2.6517 \\
\hline & $\mathrm{B}$ & 0.9999999996825778 & $7.6514 \mathrm{e}-11$ & $1.2443 \mathrm{e}-10$ & 2.3213 & 116.2732 & 1.6288 \\
\hline & $\mathrm{C}$ & 0.9999999999590754 & $1.0063 \mathrm{e}-11$ & $1.65680 \mathrm{e}-11$ & 0.4113 & 115.8890 & 3.8695 \\
\hline \multirow[t]{3}{*}{ WCPE (MASS) } & A & 0.99999999984530186 & $3.7533 \mathrm{e}-11$ & $5.6396 \mathrm{e}-11$ & 0.8467 & 115.9398 & 2.6970 \\
\hline & $\mathrm{B}$ & 0.999999999806429063 & $3.3201 \mathrm{e}-11$ & $1.1911 \mathrm{e}-10$ & 2.3218 & 116.2732 & 1.6287 \\
\hline & $\mathrm{C}$ & 0.9999999999082826 & $2.7677 \mathrm{e}-11$ & $3.8922 \mathrm{e}-11$ & 0.5858 & 117.5860 & 3.2425 \\
\hline \multirow{2}{*}{ WCPE (STATS4) } & $\mathrm{B}$ & 0.999999999833797504 & $3.5832 \mathrm{e}-11$ & $9.3068 \mathrm{e}-11$ & 2.3215 & 116.2732 & 1.6288 \\
\hline & $\mathrm{C}$ & 0.999999999951708 & $1.1129 \mathrm{e}-11$ & $2.1533 \mathrm{e}-11$ & 0.8518 & 116.3275 & 2.5979 \\
\hline \multirow[t]{3}{*}{ SPW } & A & 0.9999999999999848 & $3.9968 \mathrm{e}-15$ & $6.2258 \mathrm{e}-15$ & 1.0834 & 116.2212 & 2.3842 \\
\hline & $\mathrm{B}$ & 0.9999999999999820 & $3.9968 \mathrm{e}-15$ & $8.0151 \mathrm{e}-15$ & 2.3215 & 116.2732 & 1.6288 \\
\hline & $\mathrm{C}$ & 0.999999999999959 & $1.1102 \mathrm{e}-15$ & $1.9884 \mathrm{e}-15$ & 0.7106 & 116.6525 & 2.9439 \\
\hline \multirow[t]{2}{*}{ HTWE } & A & 0.9999999999433389 & $1.4085 \mathrm{e}-11$ & $2.3787 \mathrm{e}-11$ & 1.0000 & 116.1443 & 2.4816 \\
\hline & $\mathrm{B}$ & 0.99999999999999467 & $1.11022 \mathrm{e}-15$ & $2.7760 \mathrm{e}-15$ & 0.3582 & 116.2732 & 4.1462 \\
\hline $\operatorname{HTWE}(\mathrm{w}=1)$ & & 0.999999999629764602 & $9.2224 \mathrm{e}-11$ & $1.4834 \mathrm{e}-10$ & 2.3233 & 116.2733 & 1.6281 \\
\hline
\end{tabular}

Table 2. Summary of Comparison and Analysis of Estimates for Real Data.

HTWE $=$ Horvitz-Thompson Weighted Estimator $($ HTWE); SPW = Semi parametric Weighted Estimator,

$\mathrm{WCPE}=$ Weighted Conditional semi parametric Estimator., HTWE $(\mathrm{w}=1)=$ Un-weighted HTWE Estimator,

Var1 = Variance of HTWE Option A, Var2 = Variance of another Estimator. 
The results in Table 2 show that all estimators whose relative efficiency is greater than one are more efficient than HTWE (A) for real data for $\mathrm{n}=15$.

Table 3. Summary of the Performance of Estimators based on Bias and Standard Error for Real Data.

\begin{tabular}{|c|c|c|c|c|c|c|c|c|c|c|c|}
\hline \multirow{2}{*}{ Estimators } & \multirow{2}{*}{ Plan } & \multicolumn{2}{|l|}{$\widehat{\beta}_{0}$} & \multicolumn{2}{|l|}{$\widehat{\beta}_{1}$} & \multicolumn{2}{|l|}{$\widehat{\boldsymbol{\beta}}_{2}$} & \multicolumn{2}{|l|}{$\widehat{\boldsymbol{\beta}}_{3}$} & \multicolumn{2}{|l|}{$\widehat{\boldsymbol{\beta}}_{4}$} \\
\hline & & Bias & Error & Bias & Error & Bias & Error & Bias & Error & Bias & Error \\
\hline \multirow[t]{2}{*}{ WCPE (MOM) } & $\mathrm{A}$ & -21.60857 & $1.4995 \mathrm{e}-07$ & 0.21271 & $2.5080 \mathrm{e}-10$ & -0.01046 & $4.7754 \mathrm{e}-11$ & 0.06382 & $1.5103 \mathrm{e}-10$ & -0.01963 & $1.6181 \mathrm{e}-07$ \\
\hline & B & 26.61126 & $7.0506 \mathrm{e}-08$ & 0.08192 & $2.9014 \mathrm{e}-10$ & -0.01491 & $7.1071 \mathrm{e}-12$ & 0.01473 & $8.0998 \mathrm{e}-11$ & -0.01984 & $2.5863 \mathrm{e}-12$ \\
\hline \multirow[t]{3}{*}{ WCPE (MASS) } & A & -21.60916 & $8.3168 \mathrm{e}-08$ & 0.18817 & $3.3363 \mathrm{e}-10$ & -0.01035 & $8.5216 \mathrm{e}-12$ & 0.06680 & $8.76977 \mathrm{e}-11$ & -0.01892 & $2.7146 \mathrm{e}-12$ \\
\hline & B & 26.62450 & $6.0548 \mathrm{e}-08$ & 0.08189 & $2.2084 \mathrm{e}-10$ & -0.01491 & $6.7780 \mathrm{e}-12$ & 0.01472 & $6.5860 \mathrm{e}-11$ & -0.01984 & $1.9245 \mathrm{e}-12$ \\
\hline & $\mathrm{C}$ & -21.60980 & $8.8651 \mathrm{e}-08$ & 2.89294 & $2.8204 \mathrm{e}-10$ & $-8.9177 \mathrm{e}-03$ & $9.8664 \mathrm{e}-12$ & 0.05403 & $1.0917 \mathrm{e}-10$ & -0.01943 & $3.4142 \mathrm{e}-12$ \\
\hline \multirow[t]{2}{*}{ WCPE (STATS4) } & A & -21.60903 & $5.5451 \mathrm{e}-08$ & 0.17072 & $1.6534 \mathrm{e}-10$ & -0.01002 & $6.7411 \mathrm{e}-12$ & 0.06919 & $7.1569 \mathrm{e}-11$ & -0.01877 & $2.5745 \mathrm{e}-12$ \\
\hline & $\mathrm{C}$ & -21.60879 & $4.6955 \mathrm{e}-08$ & 0.20427 & $1.6957 \mathrm{e}-10$ & -0.01102 & $5.1655 \mathrm{e}-12$ & 0.06645 & $5.7165 \mathrm{e}-11$ & -0.01955 & $1.7770 \mathrm{e}-12$ \\
\hline \multirow[t]{3}{*}{ SPW } & A & -21.60885 & $7.7450 \mathrm{e}-10$ & 0.14864 & $2.5578 \mathrm{e}-12$ & -0.01118 & $9.2353 \mathrm{e}-14$ & 0.07652 & $9.3822 \mathrm{e}-13$ & -0.01929 & $2.9830 \mathrm{e}-14$ \\
\hline & B & 26.61728 & $4.9624 \mathrm{e}-10$ & 0.08191 & $1.8309 \mathrm{e}-12$ & -0.01491 & $5.8573 \mathrm{e}-14$ & 0.01473 & $6.8968 \mathrm{e}-13$ & -0.01984 & $2.3004 \mathrm{e}-14$ \\
\hline & $\mathrm{C}$ & -21.60913 & $3.8547 \mathrm{e}-04$ & 0.23908 & $1.1342 \mathrm{e}-06$ & $-9.9980 e-03$ & $1.3266 \mathrm{e}-08$ & 0.05820 & $4.5258 \mathrm{e}-07$ & -0.01946 & $2.0363 \mathrm{e}-14$ \\
\hline HTWE & A & -68.33828 & $4.4372 \mathrm{e}-08$ & 0.58190 & $1.6172 \mathrm{e}-10$ & -0.02928 & $5.2694 \mathrm{e}-12$ & 0.07680 & $5.6618 \mathrm{e}-11$ & 0.01894 & $1.9212 \mathrm{e}-12$ \\
\hline $\operatorname{HTWE}(\mathrm{w}=1)$ & & 26.75116 & $7.48091 \mathrm{e}-08$ & 0.08164 & $3.0336 \mathrm{e}-10$ & -0.01492 & $7.7339 \mathrm{e}-12$ & 0.01457 & $8.8192 \mathrm{e}-11$ & -0.01984 & $3.3560 \mathrm{e}-12$ \\
\hline
\end{tabular}

The simulated standard error and bias for most of the estimator are lower than for the Horvitz-Thompson weighted estimator option A, except for HTWE $\mathrm{W}=1$.

Table 4. Summary of the Performance of Estimators based on Standardized (Beta) Regression Coefficients.

\begin{tabular}{|c|c|c|c|c|c|c|}
\hline Estimators & Plan & $\widehat{\boldsymbol{\beta}}_{0}$ & $\widehat{\beta}_{1}$ & $\widehat{\boldsymbol{\beta}}_{2}$ & $\widehat{\boldsymbol{\beta}}_{3}$ & $\widehat{\boldsymbol{\beta}}_{4}$ \\
\hline \multirow[t]{2}{*}{ WCPE (MOM) } & A & $-4.2317 \mathrm{e}-15$ & $1.0510 \mathrm{e}+00$ & $8.3729 \mathrm{e}-01$ & $6.5699 \mathrm{e}-01$ & $5.2401 \mathrm{e}-01$ \\
\hline & $\mathrm{B}$ & $-1.6718 \mathrm{e}-15$ & $8.2424 \mathrm{e}-01$ & $1.2161 \mathrm{e}-01$ & $-2.0638 \mathrm{e}-01$ & $7.2585 \mathrm{e}-01$ \\
\hline \multirow[t]{3}{*}{ WCPE (MASS) } & A & $1.5269 \mathrm{e}-15$ & $9.3284 \mathrm{e}-01$ & $8.4169 \mathrm{e}-01$ & $6.9271 \mathrm{e}-01$ & $7.6632 \mathrm{e}-01$ \\
\hline & $\mathrm{B}$ & $-3.3346 e-15$ & $8.2408 \mathrm{e}-01$ & $1.2133 \mathrm{e}-01$ & $-2.0675 e-01$ & $7.2581 \mathrm{e}-01$ \\
\hline & $\mathrm{C}$ & $-1.1667 \mathrm{e}-15$ & $9.9878 \mathrm{e}-01$ & $9.0068 \mathrm{e}-01$ & $4.0943 \mathrm{e}-01$ & $4.8786 \mathrm{e}-01$ \\
\hline \multirow[t]{3}{*}{ WCPE (STATS4) } & A & $4.4415 \mathrm{e}-16$ & $8.6397 \mathrm{e}-01$ & $9.0014 \mathrm{e}-01$ & $7.3247 \mathrm{e}-01$ & $8.2153 \mathrm{e}-01$ \\
\hline & $\mathrm{B}$ & $1.7106 \mathrm{e}-15$ & $8.2407 \mathrm{e}-01$ & $1.2134 \mathrm{e}-01$ & $-2.0677 e-01$ & $7.2581 \mathrm{e}-01$ \\
\hline & $\mathrm{C}$ & $-2.8381 \mathrm{e}-15$ & $1.0369 \mathrm{e}+00$ & $7.5675 \mathrm{e}-01$ & $7.1347 \mathrm{e}-01$ & $5.7631 \mathrm{e}-01$ \\
\hline \multirow[t]{3}{*}{ SPW } & A & $6.1736 \mathrm{e}-16$ & $8.7213 e-01$ & $7.9371 \mathrm{e}-01$ & $9.5627 \mathrm{e}-01$ & $7.1912 \mathrm{e}-01$ \\
\hline & $\mathrm{B}$ & $1.0540 \mathrm{e}-16$ & $8.2416 \mathrm{e}-01$ & $1.2149 \mathrm{e}-01$ & $-2.0655 \mathrm{e}-01$ & $7.2584 \mathrm{e}-01$ \\
\hline & $\mathrm{C}$ & $-2.9349 e-15$ & $1.0456 \mathrm{e}+00$ & $8.2519 \mathrm{e}-01$ & $5.1056 \mathrm{e}-01$ & $5.2668 \mathrm{e}-01$ \\
\hline \multirow{2}{*}{ HTWE } & B & $-1.0039 \mathrm{e}-15$ & $1.0630 \mathrm{e}+00$ & $4.0809 \mathrm{e}-01$ & $1.9436 \mathrm{e}-01$ & $4.9729 \mathrm{e}-01$ \\
\hline & $\mathrm{C}$ & $-9.1776 \mathrm{e}-16$ & $1.0718 \mathrm{e}+00$ & $6.1968 \mathrm{e}-01$ & $5.2272 \mathrm{e}-01$ & $5.3370 \mathrm{e}-01$ \\
\hline HTWE $(\mathrm{w}=1)$ & & $-5.2853 e-15$ & $8.2268 \mathrm{e}-01$ & $1.1821 \mathrm{e}-01$ & $-2.1060 \mathrm{e}-01$ & $7.2512 \mathrm{e}-01$ \\
\hline
\end{tabular}

Simulated Standardized (Beta) Regression Coefficients for each coefficient for over 10,000 repeated simulations.

A standardized beta coefficient compares the strength of the effect of each individual independent variable to the dependent variable. The higher the absolute value of the beta coefficient, the stronger the effect, here we make comparison with HTWE option A.

Additional techniques of variance estimation can be found in [6].

\section{Conclusion}

In this paper, we propose two estimators for regression coefficients for analyses: an SPW estimator and a WCPE estimator. The estimators proposed provide an alternative to the conventionally used weighted (Horvitz-Thompson) estimator that can be very inefficient when applied to data with highly variable weights [4].

The two proposed estimators show significant improvement in efficiency and can be readily applied to the real data. The WCPE estimator and its standard errors can be computed using existing $\mathrm{R}$ software [16]. Therefore, its implementation is straightforward and requires only knowledge of the individual values of the weights and covariates observed in the sample.

The SPW estimator can be sensitive to the misspecification of the selection model, and hence it may not be appropriate for the analyses in which the selection model cannot be specified and estimated accurately. Also, unlike the WCPE estimator, computing the SPW estimator requires additional programming to incorporate the selection model into the estimating equations.

In survey research, regression and post stratification calibration of the sample weights are used in weighted estimation [3,9] to reduce the variance and bias (such as from deficient coverage of the sample frame) of the weighted estimators. Both types of calibration require knowledge of 
population proportions or means of design variables that are often available from population census data. In this paper, the sample weights we used were calibrated to real data categories.

To summarize, we recommend using the WCPE estimator because our simulations show it to be nearly as efficient as the SPW estimator. We recommend that the choice of the adjusted sample weights provided for analyses should be made without regard to any specific analysis but according to a general criteria of efficiency such as the $\mathrm{CV}$ of the weights. This will avoid choosing adjusted sample weights that provide the 'desired' results for a particular analysis. Further empirical research would be useful to investigate the finite sample properties of these estimators under other sample designs and types of sample weighting. Also, it would be useful to further extend the SPW and WCPE estimations to other types of studies. It is now evident that weighted regression is a viable option for obtaining estimators [19-21].

\section{References}

[1] Scott A. J, Wild CJ. Fitting logistic models under case-control or choice based sampling. JRSS, B 1986; 48: 170-182.

[2] Graubard B. I, Fears TR, Gail MH. Effects of cluster sampling on epidemiologic analysis in population-based case-control studies. Biometrics 1989; 45: 1053-1071.

[3] Korn EL, Graubard BI. Analysis of Health Surveys. Wiley: New York, NY, 1999.

[4] Scott AJ, Wild CJ. Population-based case-control studies. In Handbook of Statistics: Sample Surveys: Inference and Analysis, Vol. 29B, Pfefferman D, Rao CR (eds). Elsevier: Amsterdam, 2009; 431-453.

[5] Li Y, Graubard BI, DiGaetano R. Weighting methods for population-based case-control studies with complex sampling. JRSS (C) 2011; 60: 165-185.

[6] Rust KF, Rao JNK. Variance estimation for complex surveys using replication techniques. Statistical Methods in Medical Research 1996; 5: 283-310.

[7] Pfeffermann D, Sverchkov M. Inference under informative sampling. In Handbook of Statistics: Sample Surveys: Inference and Analysis, Vol. 29B, Pfefferman D, Rao CR (eds). Elsevier: Amsterdam, 2009; 455-487.

[8] Robins J. M, Rotnitzky A, Zhao L-P. Estimation of regression coefficients when some regressors are not always observed. Journal of American Statistical Association 1994; 89: 846866.

[9] Lumley T, Shaw PA, Dai JY. Connections between survey calibration estimators and semiparametric models for incomplete data. International Statistical Review 2011; 97 (2): 200-220.

[10] Pfeffermann D, Sverchkov M. Parametric and semiparametric estimation of regression models fitted to survey data. Sankhya, Series B 1999; 61: 166-186.

[11] Pfeffermann D, Krieger A, Rinott Y. Parametric distributions of complex survey data under informative probability sampling\}. Statistica Sinica 1981; 8: 1087-1114.

[12] Gong G, Samaniego F. Pseudo maximum likelihood estimation: theory and applications. The Annals of Statistics 1981; 9: 861-869.

[13] Pfeffermann D, Sverchkov M. Fitting generalized linear models under informative sampling. In Analysis of Survey Data, Skinner C, Chambers R (eds). Wiley: New York, 2003; 175-195.

[14] Yuan K-H, Jennrich R. Estimating equations with nuisance parameters: theory and applications. Annals of the Institute of Statistical Mathematics 2000; 52: 343-350.

[15] Krewski D., Rao J. N. K. Inference from stratified samples: properties of linearization, jackknife and balanced repeated replication methods. The Annals of Statistics 1981; 9: 1010 1019.

[16] Lumley T. Complex Surveys: A Guide to Analysis Using R. Wiley: Hoboken NJ, 2010.

[17] Landsman, V. and Graudbard, B. I. Efficient analysis of casecontrol studies. Wiley on line library.com, DOI: 10.1002/SIM $5530,2012$.

[18] J. F. Lawless, J. D. Kalbfleisch and C. J. Wild. Semiparametric Methods for Response-Selective and Missing Data Problems in Regression, Wiley, Journal of the Royal Statistical Society. Series B, Vol. 61, No. 2 (1999), pp. 413-438 (26 pages).

[19] Edwin L. Bradley. The Equivalence of Maximum Likelihood and Weighted Least Squares Estimates in the Exponential class of Distribution, Wiley, Journal of the American Statistical Association; 68: 341, 199-200, DOI (1973).

[20] Robert J. Gray. Weighted Estimating Equations for Linear Regression Analysis of Clustered Failure Time Data, Lifetime Data 9 (2): 123-138, (2003), DOI: 10.1023/A:1022932117951.

[21] Adhidev Biswas, Suman Majumder, Pratim Guha Niyogi and Aye nendranath Basu. A Weighted Likelihood Approach to Problems in Survival Data, sankhaya (2019). http://doi.org/10.1007/513571-019-00214-W

[22] Samuel J. Kamun, Richard O. Simwa and Stanley Sewe. On Derivation of the Semi-Parametric Weighted Likelihood Estimator, SPW, and the Weighted Conditional Pseudo Likelihood Estimator, WPCE, Far East Journal of Theoretical Statistics (C) 2021 Pushpa Publishing House, Prayagraj, India, http://www.pphmj.com, http://dx.doi.org/10.17654/ 